\title{
Prevalence of perinatal asphyxia and its associated factors in Ethiopia; A systematic review and meta- Analysis
}

Moges Agazhe Assemie ( $\sim$ agazhemoges@gmail.com )

Debre Markos University College of Health Science https://orcid.org/0000-0003-0380-834X

Getaye Tizazu

Debre Markos University College of Health Science

Busha Gamachu Labata

Wollega University

Alemu Simegn

Debre Markos University College of Health Science

Research article

Keywords: Birth asphyxia, perinatal asphyxia, associated factor, systematic review, Ethiopia

Posted Date: November 15th, 2019

DOI: https://doi.org/10.21203/rs.2.17346/v1

License: (c) (i) This work is licensed under a Creative Commons Attribution 4.0 International License.

Read Full License 


\section{Abstract}

Background: Perinatal asphyxia(PNA) is a severe health problem and main cause of neonatal mortality and morbidity worldwide. In Ethiopia, there are many studies conducted on PNA characterized by replete of inconsistent; unavailability of nation wide study to determines the prevalence of PNA and its determinants is an important gap. The aim of this study is to develop national consensus on pooled prevalence and associated factor key reports to enhance the quality and consistency of the evidence on perinatal asphyxia.

Method: Systematic review and meta-analysis using computerized databases; searches were performed to locate all articles on the prevalence of perinatal asphyxia. Databases included were Pub Med, Cochran library, Google Scholar, Scopus and Science Direct systematically between 2014 and April 2019. All identified studies reporting the prevalence of PNA in Ethiopia were pooled. Two independent authors extracted the data using a standardized data extraction tool. The Cochrane Q test statistics and I2 tests were used to assess the heterogeneity of the studies. Random-effects model was used to calculate pooled estimates and determinant factor of PNA in Stata/se version-14.

Result: The prevalence of PNA reported from fifteen studies was in the range of 3.1 to $47.5 \%$. The pooled occurrence of PNA in Ethiopia based on 15 articles with a sample size of 17,091 was $21.1 \%$ (95\% Cl: 14 , $28)$. There was high heterogeneity observed $(12=99.4 \%, p<0.001)$. Thus, Subgroup analysis in the study area was computed .Thus, the highest prevalence $(40.4 \%)$ was observed from Oromia region and the lowest $8 \%$ noted from Dire Dawa city administration. Pooled odd ratio estimates from included studies revealed prolonged labour $(\mathrm{OR}=3.7,95 \% \mathrm{Cl} 2.4,5.7)$, low birth weight $(\mathrm{OR}=6.5,95 \% \mathrm{Cl} 4.0,10.3)$, and Meconium stained liquor ( $\mathrm{OR}=6.6,95 \% \mathrm{Cl}$ : $4.4,10.1)$ are significant risk factors of perinatal asphyxia.

Conclusion: In this review, prolonged labour, meconium stained liquor and low birth weight are significantly associated with perinatal asphyxia. Therefore, improve facility-based intra partum care and quality neonatal resuscitation service through capacity building for health professionals is needed.

\section{Background}

Perinatal asphyxia(PNA) is a lack of blood flow or gas exchange to or from the fetus in the period immediately before, during, or after the birth process $(1,2)$. Perinatal asphyxia can result in profound systemic and neurologic sequelae due decreased blood flow and/or oxygen to a fetus or infant during the peripartum period(3). According to World Health Organization in 2017, Worldwide 2.5 million children died in the first month of life that indicate high risk of dying in the first 28 days of life, during the neonatal period(4). The incidence of PNA is higher in developing countries (Sub-Saharan Africa and Southern Asia) where limited access to maternal and neonatal care $(4,5)$.

Currently, Ethiopia is one of the top five countries contributing more than half of the neonatal deaths globally (6). Perinatal asphyxia is one of the intrapartum related common and serious clinical complications at birth and contributes significantly to most neonatal deaths, which accounts $23 \%$ 
globally $(7,8)$ and $34 \%$ in Ethiopia at the top of prematurity $(21.8 \%)$ and sepsis $(18.5 \%) .(5,9,10)$.

Perinatal asphyxia could be reduced by facility-based skilled professional intra partum care and neonatal resuscitation service $(11,12)$. In addition PNA is the cause of short and long lasting morbidity for neonates' which accounts $12.5 \%$ of overall morbidity like hypoxic ischemic organ damage followed by severe life-long pathologies, neurodegenerative diseases, mental retardation, epilepsies, and life-long functional psychotic syndromes(9).

There are many studies with inconsistent findings in Ethiopia but there is no national wide study to determine the prevalence and associated factors of birth/perinatal asphyxia is identified as a gap. Therefore, the aim of this study is to develop national consensus on pooled prevalence and associated factor key reports to enhance the quality and consistency of the evidence on perinatal asphyxia. Hence, this review could be a base line for clinicians, stakeholders, and police maker to intervene on neonatal mortality and untreatable long-term morbidity.

\section{Methods}

Study design

Systematic review and Mata-analysis using computerized databases

\section{Patient involvement}

Patients were not directly involved in the design of this study.

\section{Design settings}

Ethiopia is found in Eastern Africa bounded by Kenya to the south, Eritrea to the north, Djibouti and Somalia to the east, and Sudan to the west. Currently, the Ethiopian population is estimated to be more 106 million and approximately 3 million babies have born every year (13).

\section{Search strategy}

To find potentially relevant articles, a comprehensive search was carried out on Pub Med/MEDLINE, Google Scholar, Scopus, and Science Direct databases. We extended our search by retrieving reference lists of eligible articles, hand searches for grey literature and other relevant literature collections. The search of the literature was conducted between January and April 2019 and all studies published from April 2014 to April 2019 were considered. Search protocol was formulated by using common key words 'prevalence AND associated factor OR determinants AND birth asphyxia OR perinatal asphyxia OR neonatal asphyxia OR Neonates OR neonatal intensive care unit OR Hospitals AND Ethiopia. We presented this meta-analysis according to the scientific Preferred Reporting Items for Systematic Reviews and MetaAnalysis (PRISMA) guide line (14)

\section{Study population}


Inclusion criteria:

Study area: All studies conducted in Ethiopia

Study design: All observational study designs reporting the prevalence of perinatal asphyxia

Population: Studies involving infants immediately before, during and after birth

Language: Only articles reported in English language

Publication condition: published articles

Exclusion criteria: Articles, which were not fully accessible to assess the quality.

Outcome variables

Perinatal asphyxia and associated factors are the two main outcomes this study. The primary outcome prevalence of perinatal asphyxia was calculated as the total number of PNA divided by the total number of live birth infants in the study multiplied by 100 . The second outcome variable associated factors are determinates in the form of the log odds ratio based on binary outcomes from primary studies.

\section{Data Extraction}

All identified studies were extracted all necessary data from included articles using a standardized data extraction format adapted from the Joanna Briggs Institute (JBI), by two reviewers (GT and SA) independently. Discussions and mutual consensus were in place when possible points of view were raised. For our outcome, the data extraction format included primary author, publication year, regions of the country, study area, sample size and prevalence with $95 \%$ confidence interval.

\section{Risk of bias and quality appraisal}

The risk of bias for included studies was assessed using the JBI's critical appraisal checklist for prevalence studies (15).In addition, the included articles were evaluated for quality by two authors (BGL and $\mathrm{SA}$ ) independently and the reviewers compared their quality appraisal scores and resolved any discrepancy before calculating the final appraisal score by third authors (MAA). Only high quality studies were included in the analysis. To assess the quality of each study, the Newcastle-Ottawa Scale for crosssectional and cohort studies was adapted(16). The tool has three sections; the first section focuses on the outcome and statistical analysis of each original study with a possibility of two stars to be gained. The second section graded from five stars and considers the credibility on the methodological quality of each original study. The third section of the tool deals with the comparability of the study cases or cohorts, with a possibility of two stars to be given. Articles with a scale of $\geq 5$ out of 10 scales were considered as high quality. Consequently, all eligible studies had high quality scores.

Data processing and analysis 
Data on study design, sample size, and others were extracted in Microsoft Excel format, and then analysis was carried out using STATA/se Version 14 statistical software. Heterogeneity among reported prevalence was assessed by computing p-values of $R$-statics and Cochrane Q-test (17). The pooled prevalence of perinatal asphyxia was carried out with random effects meta-analysis model, generating the pooled $95 \%$ confidence interval by using the Der Simonian and Laird's methods. To minimize the random variations between the point estimates of the primary study; subgroup analysis was done based on study settings, study design and sample size. In addition, to identify the possible source of heterogeneity univariate metaregression was undertaken. Publication bias was assessed by visually checking for asymmetry in funnel plots and objectively testing using Egger's test at 5\% significant level. Point prevalence as well as 95\% confidence intervals were presented in the forest plot format. In this plot, the size of each box indicated the weight of the study, while each crossed line refers to $95 \%$ confidence interval.

\section{Results}

\section{PRISMA flow chart}

We retrieved 384 articles regarding prevalence of asphyxia using Pub Med, Google Scholar, Scopus, and Science Direct. Lastly, 15 studies met the eligibility criteria and included in the final meta-analysis. As shown in the follow chart of study selection process (Fig.1)

\section{Explanation for included studies}

As described in Table 1, these 15 studies were retrospective cohort and cross-sectional study design published between April 2014 and 2019. In the current meta-analysis, 17,091 study participants were represented. The prevalence of perinatal asphyxia reported was between $3.1 \%$ (18) and $47.5 \%$ (19) and the sample size of the studies ranged from 154(20) to 3684(21).

\section{Prevalence of perinatal asphyxia in Ethiopia}

Prevalence of perinatal asphyxia in Ethiopia was $21.1 \%$ (95\% Cl: 14, 28). A high heterogeneity was observed across the included papers in forest plot $\left(\mathrm{I}^{2}=99.4 \%, \mathrm{p}<0.001\right)$ (Fig. 2). As a result, a random effect meta-analysis model was computed to estimate the comprehensive prevalence of perinatal asphyxia. As described in Table 2, the region of the country where the study conducted, publication year, and sample sizes were investigated by using univariate meta-regression models to identify the possible sources of heterogeneity. However, none of the variables were found to be significant. Publication bias was assessed by funnel plot and slight presence of bias was represented substantial asymmetry (Fig.3). To confirm publication bias, objectivity Egger's test was employed and does not show the presence of small study effect $(p=0.26)$.

\section{Subgroup analysis}

In this meta-analysis, we computed subgroup analysis based on the study area/regions. Thus, the highest prevalence was determined in Oromia region $40.4 \%$ (95\% Cl: 26.1, 54.7), followed by the Tigray region 
$26.7 \%(95 \% \mathrm{Cl}: 18,35.4)$ and the lowest was noted in Dire Dawa city administration $8 \%(95 \% \mathrm{Cl}: 2,17.4)$ (Table 3).

\section{Associated factors of perinatal asphyxia in Ethiopia}

In this meta-analysis, we studied the association between perinatal asphyxia and prolonged labor by using five studies (Fig. 4). The findings from these five studies showed that the prevalence of PNA was significantly associated with long duration labour above 24 hours. Consequently, the probability of PNA was 3.7 times higher among mothers who had long duration of labour as compared to its counter parts $(\mathrm{OR}=3.7,95 \% \mathrm{Cl}: 2.4,5.7)$.

The association between meconium stained liquor and perinatal asphyxia based on studies (Fig.5) showed that the occurrence of PNA was associated with meconium stained liquor. Subsequently, the probability of PNA was 6.6 times higher among infants who had meconium stained liquor as compared to not having meconium stained liquor $(\mathrm{OR}=6.6,95 \% \mathrm{Cl}: 4.4,10.1)$.

We have done the association between low birth weight and perinatal asphyxia by using five studies (Fig.6). The findings from these articles revealed that the pooled prevalence of perinatal asphyxia was significantly associated with low birth weight. Thus, the likelihood of PNA was 6.5 times higher among infants who had low birth weight as compared to its counter parts $(\mathrm{OR}=6.5,95 \% \mathrm{Cl} 4.0,10.3)$.

\section{Discussion}

Birth asphyxia is one of the intrapartum-related common and serious clinical complications at birth. To the best of our knowledge; this meta-analysis is the first of its kind to estimate the pooled prevalence of perinatal asphyxia and determinant factor in Ethiopia.

This study revealed that, overall prevalence of perinatal asphyxia is $21 \%(95 \% \mathrm{Cl}: 14,28)$ in Ethiopia. The result of this meta-analysis is in line with a studies conducted in Gusau Nigeria (21.1\%) (33), and Dare Salaam Tanzania (21.1\%) (33).However, our finding is higher than reported by studies in Malawi (6.1\%) (34), Cameroon (8.1\%), and Iran (1\%) (16). The possible explanation for the observed variations could be attributed to methodological difference of the study, maternal and child health service, and the sample size each study used had a great impact on the prevalence of perinatal asphyxia.

We did subgroup analysis by study area/region due to a significant heterogeneity finding. Thus, highest prevalence of perinatal asphyxia was determined in Oromia region $40.4 \%$, followed by the Tigray region $26.7 \%$ and the lowest was noted in Dire Dawa city administration $8 \%$. This variation could be the differences in accessibility of delivery institution, health seeking behavior, skill of health professionals, and behavioral characteristics of caregivers.

Meconium stained liquor was found statistically associated factor with perinatal asphyxia. This finding is in line with those of studies conducted in Nigeria, England, and Thailand (35-37). In healthy, well oxygenated fetuses, this diluted meconium is readily cleared from the lungs by normal physiological 
mechanism. However, the Presence of meconium in the amniotic fluid might be lead to aspiration of meconium into the lung or air way obstruction that could eventually result in perinatal asphyxia.

In addition, prolonged labor was a risk factor for perinatal asphyxia. This result is consistent with the findings in India, Cameron, Pakistan, Nigeria, and Thailand $(35,38-41)$. This could be due to the prolonged labor can associated with fetal and maternal exhaustion and fetal distress and/or will be exposed to Pitocin, forceps/vacuum extractors which could be a cause for the baby to have PNA.

The other determinant factor of perinatal asphyxia is low birth weight. This study is congruent to the studies reported in Nepal, Thailand, Uganda and England $(35,37,39,42)$. The possible justification could be low-birth-weight infants often have pulmonary immaturity and limited respiratory muscle strength which will cause difficulty of birthing and cardiopulmonary transition(43). In contrary, high birth weight at birth (a proxy for birth weight) had been found an associated factor for birth asphyxia (44)

\section{Strength and Limitations of the study}

Critical appraisal by three authors is the strength of the study. While the first limitation of the study was only English reports were considered to conduct this study. Some of the studies included in this review had a relatively small sample size. Besides, this meta-analysis represented only studies reported from five regions and two administrative town of the country.

\section{Conclusion}

In this review, pooled perinatal asphyxia in Ethiopia was found high. Low birth weight, prolonged labor and meconium stained liquor are risk factor for PNA. Improve facility-based intra partum care and neonatal resuscitation service quality through capacity building for health professionals.

\section{Abbreviations}

APGAR

Appearance Pulls Grimes Activity Respiration

$\mathrm{Cl}$

Confidence Interval

EDHS

Ethiopian Demography and Health Survey

$\mathrm{JBI}$

Joanna Briggs Institute 
OR

Odd Ratio

PNA

Perinatal Asphyxia

Declarations

\section{Ethics approval and consent to participate}

Not applicable

\section{Consent for publication}

Not applicable

\section{Availability of data and material}

Minimal data could be accessed upon request from first author (MAA)

\section{Competing interests}

The authors declare that they have no competing interests

\section{Funding}

Not applicable

\section{Author contributions}

MAA: Conception of research protocol, study design, literature review, data extraction, data analysis, interpretation and drafting the manuscript.

GT: Study design and contribute on data extraction, quality assessment, data analysis and manuscript review

BGL: contribute on data extraction, quality assessment, data analysis and manuscript review 
SA: Study design, literature review, data extraction, data analysis, interpretation and manuscript review. All authors have read and approved the manuscript

\section{Acknowledgements}

Authors of primary study

\section{Author's information}

${ }^{1}$ Lecturer of Biostatistics under department of Public Health, College of Health Sciences, Debre Markos University, Debre Markos, Ethiopia.

${ }^{2}$ Lecturer of Health informatics under department of Health informatics, College of Health Sciences, Debre Markos University, Debre Markos, Ethiopia

${ }^{3}$ Lecturer of clinical pharmacy under department of Pharmacy, Wollega University, Nekemte, Ethiopia

${ }^{4}$ Lecturer of Epidemiology under department of Public Health, College of Health Sciences, Debre Markos University, Debre Markos, Ethiopia.

\section{Reference}

1.JE Lawn ZC. 4 million neonatal deaths: when? Where? Why?. The lancet. 2005.

2.WHO. World health statistics. 2013.

3.UNIAGCME. United Nations Inter-agency Group for Child Mortality Estimation 2018.

4.WHO. world Health Organization Key facts Sheet. 2018

5.unicef. Maternal and Newborn Health Disparities in Ethiopia. 2015.

6.UNICEF WB, UNFPA,. Levels \& Trends in child mortality: Report. 2017.

7.Lopez M. Measuring the global burden of disease and epidemiological transitions (2002-2030) Trop Med Parasitol 2006.

8.WHO U, WB,. WHO,UNICEF and WB population Division Levels and trends in child mortality. 2013.

9.AG Demisse FA, Gizaw MA, Tigabu Z. Patterns of admission and factors associated with neonatal mortality among neonates admitted to the neonatal intensive care unit of University of Gondar Hospital, Northwest Ethiopia. Dove press. 2017. 
10.ND Binyam NA, Damte TD, Hordofa LO. Neonatal Mortality and its risk factors in Eastern Ethiopia:. Epidemiology Biostatistics and Public Health 2016;13.

11.SK Lawn SCea. No cry at birth: global estimates of intrapartum stillbirths and intrapartum- related neonatal deaths. Bull World Health Organ. 2005.

12.Ayantu Fekede. Knowledge, attitude and practice towards management of birth asphyxia among nurses and midwives working in labour ward and NICU in governmental hospitals, Addis Ababa, Ethiopia,. 2017.

13.Ethiopia Population (LIVE) worldometers http://www.worldometers.info/worldpopulation/ethiopiapopulation/.

14.Liberati A AD, Tetzlaff J, Mulrow C, et.al. The PRISMA statement for reporting systematic reviews and meta-analyses of studies that evaluate health care interventions: explanation and elaboration. $\mathrm{J}$ Clin Epidemiol. 2009.

15.Z Munn SM, D Riitano, K Lisy,. The development of a critical appraisal tool for use in systematic reviews addressing questions of prevalence.. International journal of health policy and management. 2014.

16.Chikuse B CE, Maluwa A, Malata A, Odland J (2012) Midwives' adherence to guidelines on the management of birth asphyxia in Malawi. Open J Nurs 2: 351-357.

17.Rücker G SG, Carpenter JR, Schumacher M:. Undue reliance on I 2 in assessing heterogeneity may mislead. BMC Med Res Methodol 2008.

18. Neil Abdurashid Ibrahim AM, Selah Abdulie,. Prevalence of Birth Asphyxia and Associated Factors among Neonates Delivered in Dilchora Referral Hospital, in Dire Dawa, Eastern Ethiopia. Clinics in Mother and Child Health. 2017.

19.Gurmesa Tura Debelew MFA, Alemayehu Worku Yalew,. Determinants and Causes of Neonatal Mortality in Jimma Zone, Southwest Ethiopia: A Multilevel Analysis of Prospective Follow Up Study. Plose one. 2014.

20. Worku Necho Asfere AY. Neonatal asphyxia and associated factors among neonates on labor ward at debre-tabor general hospital, Debre Tabor Town, South Gonder, North Centeral Ethiopia. International Journal of Pregnancy \& Child Birth. 2018.

21.Berhe Weldearegawi YAM, Semaw Ferede Abera, Yemane Ashebir, Fisaha Haile,Afework Mulugeta, Frehiwot Eshetu, and Mark Spigt,. Infant mortality and causes of infant deaths in rural Ethiopia. BMC Public health. 2015. 
22.Abdifatah Elmi Farah AHA, Ahmed Tahir Ahmed,. Trends of admission and predictors of neonatal mortality: A hospital based retrospective cohort study in Somali region of Ethiopia. Plose one. 2018.

23. Tujare Tunta Orsido NAA, Tezera Moshago Berheto. Predictors of Neonatal mortality in Neonatal intensive care unit at referral Hospital in Southern Ethiopia: a retrospective cohort study. BMC Pregnancy and Childbirth. 2019.

24.Roba AA DD. Morbidities, Rate and Time Trends of Neonatal Mortality in Dilchora Referral Hospital,Dire Dawa, Ethiopia, 2012-2017. Austin Medical Sciences. 2017.

25.Gdiom Gebreheat TT, Dessalegn Kiros, Hirut Teame,Natnael Etsay,Guesh Welu,and Desta Abraha,. Prevalence and Associated Factors of Perinatal Asphyxia among Neonates in General Hospitals of Tigray, Ethiopia, 2018. BioMed Research International. 2018.

26.Zelalem Jebessa Wayessa TB, Jophin Joseph,. Birth asphyxia and associated factors among newborns delivered in Jimma zone public hospitals, Southwest Ethiopia: A cross-sectional study. J Midwifery Reprod Health. 2018.

27. Hayelom Gebrekirstos Mengesha BWS. Cause of neonatal deaths in Northern Ethiopia: a prospective cohort study. BMC Public health. 2017.

28.Abayneh Girma Demisse, Fentahun Alemu, Mahlet Abayneh Gizaw, Zemene Tigabu. Patterns of admission and factors associated with neonatal mortality among neonates admitted to the neonatal intensive care unit of University of Gondar Hospital, Northwest Ethiopia. Dove press. 2017.

29.Gudayu TW. Proportion and factors associated with low fifth minute Apgar score among singleton newborn babies in Gondar University referral hospital; North West Ethiopia. African Health Sciences. 2017.

30. Mehretie Kokeb TD. Institution beased prospective cros sectional study on patterens of Neonatal morbidity at Gondar university hospital neonatal unit,northwest Ethiopia. Ethiop J Health sci. 2016.

31. Ayenew Engida Yismaw AAT. Proportion and factors of death among preterm neonates admitted in University of Gondar comprehensive specialized hospital neonatal intensive care unit, Northwest Ethiopia. BMC research notes. 2018.

32.Birhanu Wondimeneh Demissie BBA, Tesfaye Yitna Chichiabellu, Feleke Hailemichael Astawesegn,. Neonatal hypothermia and associated factors among neonates admitted to neonatal intensive care unit of public hospitals in Addis Ababa, Ethiopia. BMC Pediatrics. 2018.

33.Kali GT M-BM, Van Zyl J, Smith J, Rutherford M (2015) Management of therapeutic hypothermia for neonatal hypoxic ischaemic encephalopathy in a tertiary centre in South Africa. Arch Dis Child Fetal Neonatal Ed 100: F519-F523. 
34.Boskabadi H AF, Doosti H, Zakerihamidi M (2015) Assessment of Risk Factors and Prognosis in Asphyxiated Infants. Iran J Pediatr 25: e2006.

35.C Pitsawong PP. Risk factors associated with birth asphyxia in Phramongkutklao Hospital.. Thai Journal of Obstetrics and Gynecology. 2012.

36.S Thangwijitra CS, S Kanchanawat. Risk factors of birth asphyxia in pregnancy 37 complete weeks and over by Apgar score less than 7 at 5 minutes. Thai Journal of Obstetrics and Gynecology. 2012.

37.M Martinez-Biarge, J Diez-Sebastian, CJ Wusthoff, E Mercuri, FM Cowan. Antepartum and intrapartum factors preceding neonatal hypoxic-ischemic encephalopathy.. Pediatrics. 2013.

38.P Foumane GN, ET Mboudou,, JD Sama SN, B Moifo,. Risk factors of clinical birth asphyxia and subsequent newborn death following nuchal cord in a low-resource setting.. Open Journal of Obstetrics and Gynecology. 2013.

39.AC Lee LM, JM Tielsch, et al. Risk factors for neonatal mortality due to birth asphyxia in southern Nepal: a prospective, community-based cohort study. Pediatrics. 2008.

40.AD Cunha DF, PF Melo, et al. Factors associated with perinatal asphyxia.. Revista Brasileira de Ginecologia e Obstetrícia 2004.

41.S Berglund. Severe asphyxia due to substandard care during labor. Department of Clinical Science and Education. 2010.

42.B Jagkaew. Factors associated with birth asphyxia in Fang Hospital. Mahasarakham Hospital Journal. 2014.

43.Mandy G. Small for gestational age infant: complications. 2011.

44.F Tabassum AR, S Ariff, S Soofi, ZA Bhutta,. Risk Factors Associated with Birth Asphyxia in Rural District Matiari, Pakistan: A Case Control Study. International Journal of Clinical Medicine. 2014;vol. 05.

\section{Tables}

Table1: descriptive summary of studies included in the systematic review and meta-analysis in the prevalence of perinatal asphyxia in Ethiopia, 2019 


\begin{tabular}{|c|c|c|c|c|c|c|}
\hline $\begin{array}{l}\text { uthorship } \\
\text { ieference) }\end{array}$ & Publication(yr) & $\begin{array}{l}\text { Study } \\
\text { Area/ } \\
\text { Region }\end{array}$ & Study design & $\begin{array}{l}\text { Mid- } \\
\text { Study } \\
\text { period }\end{array}$ & Sample & $\begin{array}{l}\text { Prevalence at } \\
\text { 95\%CI }\end{array}$ \\
\hline $\begin{array}{l}\text { rahim et al. } \\
\text { 8) }\end{array}$ & 2017 & $\begin{array}{l}\text { Dire } \\
\text { Dawa }\end{array}$ & Retrospective & 2015.5 & 302 & $3.1(1.2,5.1)$ \\
\hline $\begin{array}{l}\text { arah et al. } \\
\text { (2) }\end{array}$ & 2018 & Somalia & Retrospective & 2017 & 792 & $3.4(2.1,4.7)$ \\
\hline $\begin{array}{l}\text { rsido et al. } \\
\text { i3) }\end{array}$ & 2019 & SNNP* & Retrospective & 2016 & 964 & $26.2(23.5,29.0)$ \\
\hline $\begin{array}{l}\text { eldearegawi } \\
\text { al.(21) }\end{array}$ & 2015 & $\begin{array}{l}\text { Addis } \\
\text { Ababa }\end{array}$ & Retrospective & 2011 & 3684 & $13.8(12.7,14.9)$ \\
\hline $\begin{array}{l}\text { oba et al. } \\
\text { i) }\end{array}$ & 2017 & $\begin{array}{l}\text { Dire } \\
\text { Dawa }\end{array}$ & Retrospective & 2015 & 3418 & $12.7(11.6,13.9)$ \\
\hline $\begin{array}{l}\text { sfere et al. } \\
0)\end{array}$ & 2018 & Amhara & $\begin{array}{l}\text { Cross } \\
\text { sectional }\end{array}$ & 2017 & 154 & $29.9(22.7,37.1)$ \\
\hline $\begin{array}{l}\text { ebreheat et } \\
.(25)\end{array}$ & 2018 & Tigray & $\begin{array}{l}\text { Cross } \\
\text { sectional }\end{array}$ & 2018 & 423 & $22.1(18.2,26.1)$ \\
\hline $\begin{array}{l}\text { bessa et al } \\
\text { 6) }\end{array}$ & 2018 & Oromiya & $\begin{array}{l}\text { Cross } \\
\text { sectional }\end{array}$ & 2015 & 371 & $32.9(28.1,37.7)$ \\
\hline $\begin{array}{l}\text { engesha et } \\
\text {.(27) }\end{array}$ & 2017 & Tigray & $\begin{array}{l}\text { Cross } \\
\text { sectional }\end{array}$ & 2014 & 1152 & $31(28.3,33.7)$ \\
\hline $\begin{array}{l}\text { emisse et al. } \\
\text { (8) }\end{array}$ & 2017 & Amhara & $\begin{array}{l}\text { Cross } \\
\text { sectional }\end{array}$ & 2015.5 & 769 & $12.5(10.5,14.8)$ \\
\hline $\begin{array}{l}\text { ebelew et al. } \\
9)\end{array}$ & 2014 & Oromiya & $\begin{array}{l}\text { Cross } \\
\text { sectional }\end{array}$ & 2012.5 & 3604 & $47.5(45.9,49.1)$ \\
\hline $\begin{array}{l}\text { udayu et al. } \\
\text { 9) }\end{array}$ & 2017 & Amhara & $\begin{array}{l}\text { Cross } \\
\text { sectional }\end{array}$ & 2013 & 261 & $16(11.6,20.5)$ \\
\hline $\begin{array}{l}\text { ehretie et } \\
\text { (30) }\end{array}$ & 2016 & Amhara & $\begin{array}{l}\text { Cross } \\
\text { sectional }\end{array}$ & 2014 & 325 & $16.6(12.6,20.7)$ \\
\hline $\begin{array}{l}\text { smaw et al. } \\
\text { i1) }\end{array}$ & 2018 & Amhara & $\begin{array}{l}\text { Cross } \\
\text { sectional }\end{array}$ & 2017 & 516 & $26.6(22.7,30.4)$ \\
\hline $\begin{array}{l}\text { emissie et } \\
\text {.(32) }\end{array}$ & 2018 & $\begin{array}{l}\text { Addis } \\
\text { Ababa }\end{array}$ & $\begin{array}{l}\text { Cross } \\
\text { sectional }\end{array}$ & 2016 & 356 & $23.6(19.2,28.0)$ \\
\hline
\end{tabular}

SNNP*=Southern Nation, Nationality and People

Table 2: Related factors with heterogeneity of perinatal asphyxia prevalence (based on univariate meta-regression) 


\begin{tabular}{|l|l|l|}
\hline Variables & Coefficient & P-value \\
\hline Publication year & -0.23 & 0.51 \\
\hline Sample size & 0.005 & 0.32 \\
\hline Region \\
\hline Amhara & -4.1 & 0.61 \\
\hline Addis Ababa & -0.011 & 0.88 \\
\hline Oromia & -5.2 & 0.24 \\
\hline Dire Dawa & -5.3 & 0.51 \\
\hline SNNP \&Somalia & -3.2 & 0.6 \\
\hline Tigray & -0.032 & 0.79 \\
\hline
\end{tabular}

Table 3.Subgroup analysis of perinatal asphyxia in Ethiopia, 2019 ( $\mathrm{n}=15)$

\begin{tabular}{|c|c|c|c|c|c|}
\hline Variables & Characteristics & $\begin{array}{l}\text { No-articles } \\
\text { included }\end{array}$ & $\begin{array}{l}\text { Sample } \\
\text { size }\end{array}$ & $\begin{array}{l}\text { Prevalence with }(95 \% \\
\text { CI) }\end{array}$ & $\overline{p \text {-value }}$ \\
\hline \multirow[t]{6}{*}{ By region } & Amahra & 5 & 2025 & $19.9(13.7,26.2)$ & $<0.001$ \\
\hline & Addis Ababa & 2 & 4040 & $18.5(8.9,20.1)$ & $<0.001$ \\
\hline & Oromia & 2 & 3975 & $40.4(26.1,54.7)$ & $<0.001$ \\
\hline & SNNP\&Somalia & 2 & 1756 & $14.8(7.6,37.1)$ & $<0.001$ \\
\hline & Dire Dawa & 2 & 3720 & $8(2,17.4)$ & $<0.001$ \\
\hline & Tigray & 2 & 1575 & $26.7(8,35.4)$ & $<0.001$ \\
\hline \multirow{2}{*}{$\begin{array}{l}\text { Study } \\
\text { design }\end{array}$} & Retrospective & 5 & 9160 & $11.8(5.8,17.8)$ & $<0.001$ \\
\hline & Cross sectional & 10 & 7931 & $25.6(16.4,35.4)$ & 0.004 \\
\hline \multirow[t]{2}{*}{$\begin{array}{l}\text { Sample } \\
\text { mean }\end{array}$} & $\begin{array}{l}\text { Above } \\
\text { mean(1139) }\end{array}$ & 4 & 4558 & $26 \%(10.4,42.1)$ & 0.008 \\
\hline & $\begin{array}{l}\text { Below } \\
\text { mean(1139) }\end{array}$ & 11 & 12532 & $19.2(12.6,25.7)$ & $<0.001$ \\
\hline
\end{tabular}

\section{Figures}




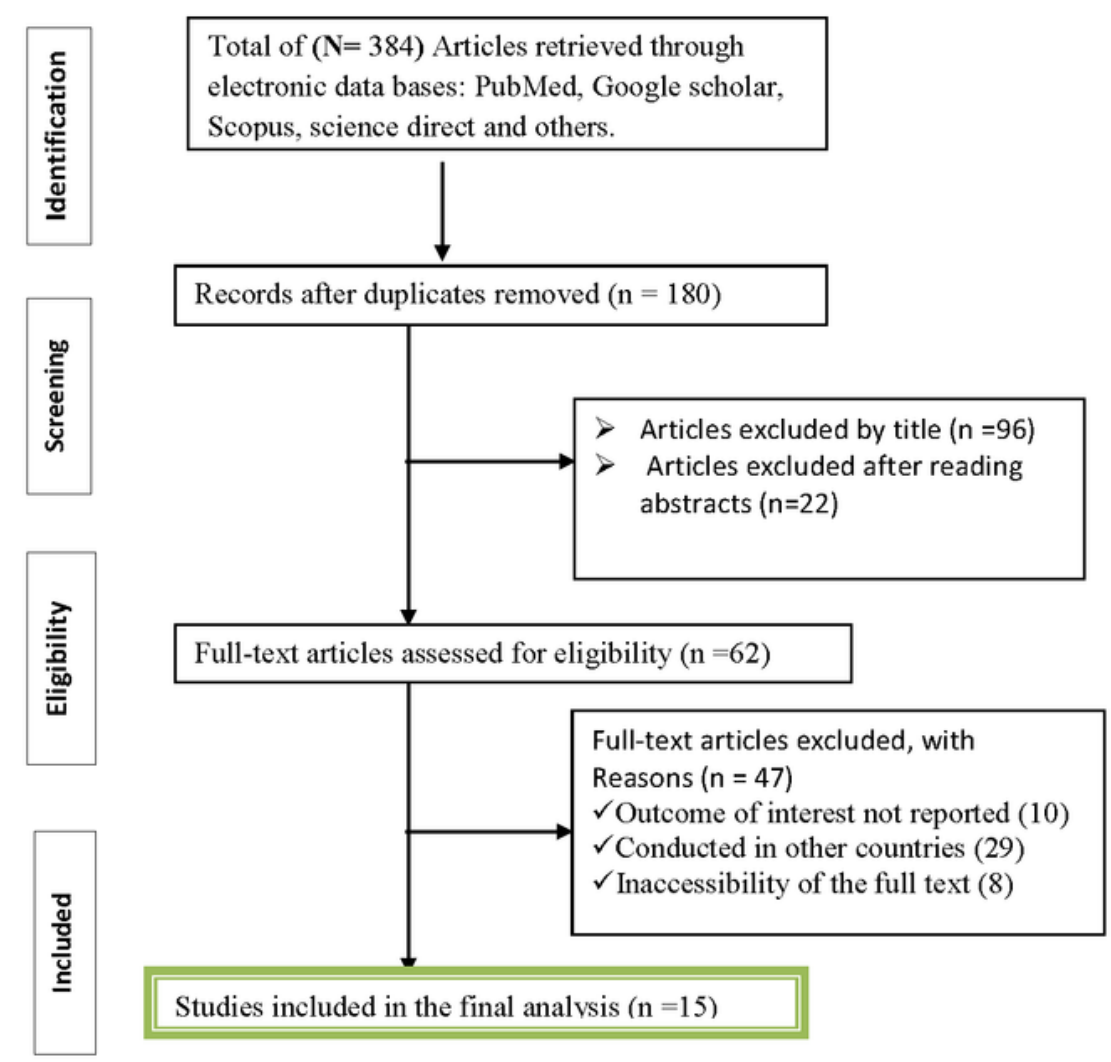

\section{Figure 1}

PRISMA study selection flow diagram for systematic review and meta-analysis on perinatal as-phyxia in Ethiopia. 


Study
ID
Ibrahim et al. (2017)
Farah et al. (2018)
Orsido et al. (2019)
Weldearegawi et al. (2015)
Asfere et al. (2018)
Gdiom et al. (2018)
Jebessa et al. (2018)
Mengesha et al. (2017)
Demisse et al. (2017)
Debelew et al. (2014)
Gudayu et al. (2017)
Merhetie et al. (2016)
Yismaw et al. (2018)
Roba et al. (2017)
Demissie et al. (2018)
Overall (I-squared = 99.4\%, p=0.000)
NOTE: Weights are from random effects analysis

\section{Figure 2}

Forest plot of the pooled prevalence of perinatal asphyxia in Ethiopia. 


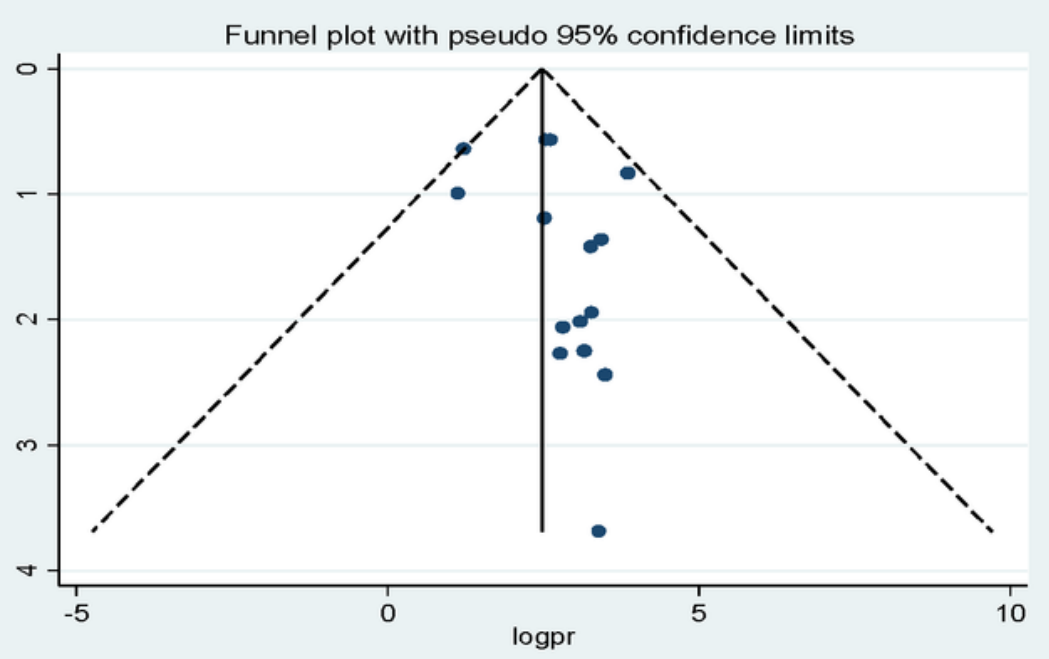

Figure 3

Funnel plot with $95 \%$ confidence limits of the pooled prevalence of perinatal asphyxia in Ethio-pia. 
Study

ID
$\mathrm{OR}(95 \% \mathrm{Cl})$

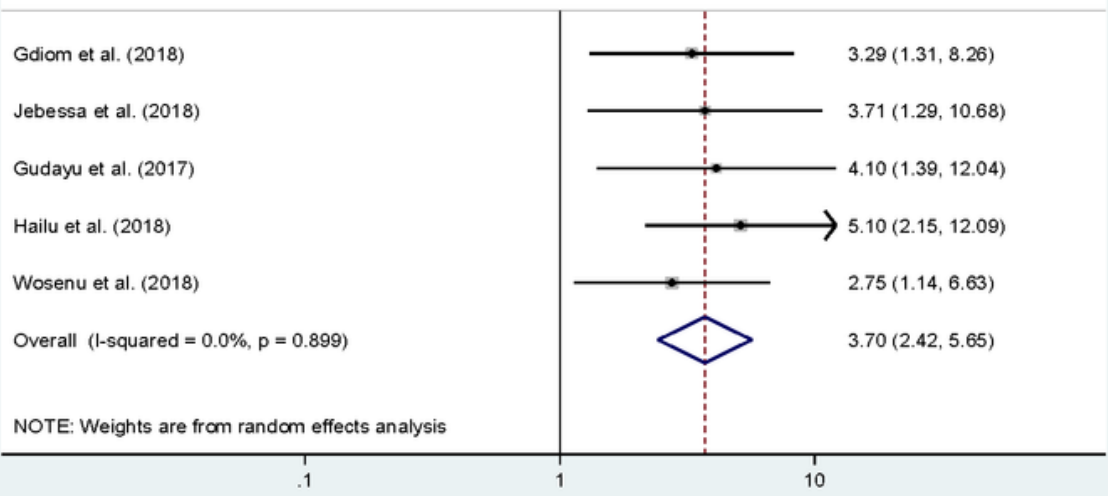

\section{Figure 4}

pooled odd ratio between perinatal asphyxia and prolonged labour 
Study

ID

$\mathrm{OR}(95 \% \mathrm{Cl})$

\begin{tabular}{l} 
Gdiom et al. (2018) \\
Jebessa et al. (2018) \\
Gudayu et al. (2017) \\
Wosenu et al. (2018) \\
Tasaw et al. (2018) \\
Overall (I-squared $=19.2 \%, \mathrm{p}=0.292)$ \\
NOTE: Weights are from random effects analysis \\
\hline .1
\end{tabular}

Figure 5

pooled odd ratio between perinatal asphyxia and meconium stained liquor 
Study

ID

OR(95\%Cl)

\begin{tabular}{l} 
Gdiom et al. (2018) \\
Jebessa et al. (2018) \\
Gudayu et al. (2017) \\
Wosenu et al. (2018) \\
Tasew et al. (2018) \\
Overall (1-squared $=0.0 \%, \mathrm{p}=0.631)$ \\
NOTE: Weights are from random effects analysis \\
\hline
\end{tabular}

Figure 6

pooled odd ratio between perinatal asphyxia and low birth weight 